\title{
Ueber eine Gattung doppelt reell periodischer Functionen
} zweier Verïnderlicher.

Von

Orto Sraude in Dorpat.

Das Umkehrproblem der hyperelliptischen Integrale 1. Gattung rom Geschlecht $p$ knüpft sich an $p$ Gleichungen von der Form:

$$
\sum_{1}^{p} \int_{u_{k}}^{x_{k}} \frac{x^{i-1} d x}{\sqrt{k i(x)}}=t_{i}, \quad i=1,2, \ldots, p,
$$

an, welche die zweimal $p$ Veränderlichen $x_{1}, x_{2}, \ldots, x_{p}$ und $t_{1}, t_{2}, \ldots, t_{p}$ mit einander verbinden und in

$$
R(x)=\left(a_{0}-x\right)\left(a_{1}-x\right)\left(a_{2}-x\right) \ldots\left(a_{2 p}-x\right)
$$

eine ganze rationale function rom $(2 p+1)^{\text {ten }}$ Grade enthalten. Das Umkehrproblem besteht in der Aufgabe, die Coefficienten derjenigen Gleichung $p^{\text {tea }}$ Grades, deren Wurzeln $x_{1}, x_{2}, \ldots, x_{p}$ sind, als Functionen von $t_{1}, t_{2}, \ldots, t_{p}$ darzustellen. Diesem mittels der Thetafunctionen von $p$ Argumenten gelösten "vollständigen "Umkehrproblem stellen sich eine Reihe anderer, "unvollständiger" Umkehrprobleme zur Seite. Das erste derselben bezieht sich auf die folgenden $p-1$ Gleichungen 2 wischen $2 w e i m a l ~ p-1$ Veränderlichen $x_{1}, x_{2}, \ldots, x_{p-1}$ und $t_{1}, t_{2}, \ldots, t_{p-1}$ :

$$
\sum_{i}^{n-1} \int_{a_{h}}^{z_{h}} \frac{x^{i-1} d x}{V / \overline{R(x)}}=t_{i}, \quad i=1,2, \ldots, p-1 ;
$$

es verlangt die rationalen symmetrischen Functionen der Variablen $x_{1}, x_{2}, \ldots, x_{p-1}$ durch die Variablen $t_{1}, t_{2}, \ldots, t_{p-1}$ darzustellen. Bin ¿weites unvollständiges Dmkehrproblem würde eine ahnliche Fragestellung an ein entsprechend weiter reducirtes system von $p-2$ 
Gleichnngeu zwischen zweimal p-2 Variablen anschliessen. Bin $(p-2)^{\text {les }}$ und $(p-1)^{\text {los }}$ Problem jener Reihe endlich geht beziehungsweise ron den Gleichungen:

$$
\begin{aligned}
& \int_{a_{1}}^{x_{1}} \frac{d x_{1}}{\sqrt{R\left(x_{1}\right)}}+\int_{a_{2}}^{x_{1}} \frac{d x_{2}}{\sqrt{\tilde{R}\left(x_{2}\right)}}=t_{1},
\end{aligned}
$$

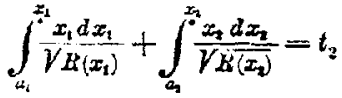

und der Gleicbung:

$$
\int_{a_{1}}^{x_{3}} \frac{d x_{1}}{\sqrt{k\left(x_{1}\right)}}=t_{1}
$$

aus und verlangt die symmetrischen functionen von $x_{1}, x_{2}$ und bezüglich die Variable $x_{1}$ durch $t_{1}, t_{2}$ und durch $t_{1}$ auszudrücken. Dabei bleibt $R(x)$ überall vom Grade $2 p+1$.

Gerade diese "unvollstïndigen" Umkehrprobleme haben für sn. wendungen auf Mechanil ein besonderes Interesse, wobei die in ihnen auftretenden Variablen im Wesentlichen auf reelle Werthe beschrïnkt werden können.

Das Problem der Umkehrung eines eincelnen byperelliptischen Integrals von beliebigem Geschlecht hat Weierstrass bohandelt*), und zwar in einer wesentlich verallgemeinerten Form. Er stellt dio Aufgabe eine eindentige, endliche und stetige Function $E(x)$ der oberen Grenze des Integrals

$$
\int_{d}^{x} \frac{d x}{\sqrt{ } F^{2}(x)}=t
$$

als Function ron $t$ zu untersuchen, wenn $F(x)$ eine gegebene eindeutige Function von $x$ ist. Nach dem Ergebniss seiner Untersuchung ist eine solche Function $E(x)$ unter gewissen Voranssetzungen uber $F(x)$ für alle reellen Werthe ron $t$ eine eindentige, stetige and endliche, sowie einfach reell periodische Function von $t$. Sie ist ferner durch eine für alle Werthe ron $t$ gleichmässig convergente trigonometrische Reihe darstellbar. Unter Beschränkung auf reelle Variable hat man hiermit eine Gattung periodischer Functionen gewonnen, welche sich als Verallgemeinerungen der Umkehrfunctionen der cyklometrischen und elliptischen Integrale darstellen.

Man kann nun ähnliche Untersuchungen, wie sie hierdurch an das letzte der oben erwähnten unrollständigen Umkehrprobleme

-) Weierstrass, Deber eine Gattung reell periodischer Functionen, Monatberichte der Konigl. Academio der Wissenschaften 2 a Berlin, 1868. 
sich angeknüpft haben, anch an die anderen unvollständigen Umkehr. probleme anschliessen. Die rorliegende Mitheilung beschäftigt sich zunächst mit dem vorletaten jener unvollständigen Umkehrprobleme, welches zugleich in wesentlich verallgemeinerter Gestalt (vgl \& 1) behandelt wird. Es wird vor Allem die Symmetrie des Ansatzes in Bezug auf die beiden Variablen $x_{1}, x_{2}$ und die Symmetrie der $z \mathrm{n}$ untersuchenden Functionen dieser Variablen aufgegeben. Die Betrachtung führt zu einer Gattong doppelt reell perjodischer Functionen zweier reeller Veränderlicher, zu welchen als specielle Fälle die hyperelliptischen Functionen zweier Variabler vom Geschlecht $p=2$ gehören.

Wie die Weierstrass'schen reell periodischen Functionen einer Veränderlichen, so finden auch die hier betrachteten doppelt reell periodischen Functionen zweier Argamente in der Mechanik eine mehrfache Verwerthung. Dabei geben diese Functionen, indem eine der Variablen $t_{1}, t_{2}$ constant gesetat wird, in bedingt periodische Functionen ( $\nabla g l . \$ 8$ ) der anderen über. Ich beabsichtige in einer späteren Arbeit anf die versehiedenen Anwendungen in der Mechanik näher einzugehen. *)

$\S 1$.

Formulirnng des za behandelnden Jmkehrproblems.

Zwischen zwei Paaren reeller Veränderlicher $x_{1}, x_{2}$ und $t_{1}, t_{2}$ sollen die Gleichungen:

bestehen.

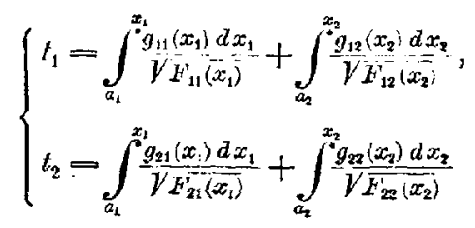

Unter $F_{11}(x), F_{21}(x), F_{12}(x), F_{22}(x)$ sind gegebene Functionen von $x$ zu verstehen, von denen die beiden ersten für zwei reelle Werthe $x=a_{1}, x=b_{1}$ und die beiden letaten für zwei reelle Werthe $x=a_{2}$, $x=b_{3}$ verschwinden. Setzt man mit Rücksicht daranf:

$$
\left\{\begin{array}{l}
F_{\alpha 1}(x)=\left(x-a_{1}\right)\left(b_{1}-x\right) f_{\alpha 1}(x), \\
F_{\alpha 2}(x)=\left(x-a_{2}\right)\left(b_{2}-x\right) f_{\alpha 2}(x),
\end{array}\right.
$$

$a=1,2$, so sollen $f_{\alpha 1}(x)$ in dem Intervalle $a_{1}<x<b_{1}$ und $f_{t 2}(x)$ in dem Intervalle $a_{2}<x<b_{2}$ eindentige und stetige Functionen von $x$ sein, daselbst einen beständig positiven reellen Werth besitzen and weder 0 noch $\infty$ werden. Zugleich sollen zwischen den Vorzeichen

*) Vgl. die vorlänfige Mittheilang: Veber bedingt periodische Bewegangen, Sitzungsberichte der Dorpater Natarforschergesellschaft, April, 1887. 
der Quadratwurzeln aus den eiuzeloen Bestandiheilen der Gleichungen (2) die Beziehungen:

$$
\sqrt{F_{a \beta}\left(x_{p}\right)}=\sqrt{\left(x_{\beta}-a_{\beta}\right)\left(b_{\beta}-x_{\beta}\right)} \sqrt{f_{\alpha \beta}\left(x_{\beta}\right)},
$$

$\alpha, \beta=1,2$, festgesetzt und unter $\sqrt{f_{\alpha \beta}\left(x_{\beta}\right)}$ der positive Worth der Quadratwurzel aus der positiven reellen Gröse $f_{\sigma \beta}\left(x_{\beta}\right)$ vexstanden sein.

Was ferner die functionen $g_{11}(x), g_{31}(x)$ und $g_{12}(x), g_{22}(x)$ un. geht, so sollen dieselben beziebungsweise in den Intervallen $a_{1}<x<b_{1}$ and $a_{2}<x<b_{2}$ eindentige und stetige Functionen von $x$ sein, div daselbst niemals jhr Vorzeichen wechseln und niemals $\infty$ werden.

Endlich soll die Determinante:

$$
D\left(x_{1}, x_{2}\right)=\frac{g_{13}\left(x_{1}\right)}{V / f_{1}\left(x_{3}\right)} \frac{g_{2 x}\left(x_{2}\right)}{V / f_{x x}\left(x_{2}\right)}-\frac{g_{11}\left(x_{1}\right)}{V / f_{2}\left(x_{1}\right)}-\frac{g_{12}\left(x_{1}\right)}{V / f_{12}\left(x_{2}\right)}
$$

für alle den Ungleichungen:

$$
a_{1}<x_{1}<b_{1}, \quad a_{2}<x_{2}<b_{2}
$$

genügenden Werthepaare $x_{1}, x_{2}$ beständig positiv oder bestindig negativ sein und fir solche Werthepaare niemals rerschwinden.

Nach diesen Voraussetzungen uber die in den Gleichungen (1) auftretenden Functionen soll eino beliebig gegebene Function der reellen Variablen $x_{1}, x_{2}: \mathscr{E}\left(x_{1}, x_{2}\right)$, welche für alle den Uogleichungen (j) genügenden Werthepaare $x_{1}, x_{2}$ eindentig, endlich und stetig ist, in ihrer Abhängigkeit von den zeelleu Variablen $t_{1} t_{2}$ unterstuclit perden. Es sei etwa:

$$
E\left(x_{1}, x_{2}\right)=G\left(t_{1}, t_{2}\right)
$$

Daneben sollen auch eindeutige Fonctionen von $x_{1}, x_{2}$ und den vies Quadratorurzeln:

$$
\sqrt{x_{1}-a_{1}}, \sqrt{b_{1}-x_{1}}, \sqrt{x_{2}-a_{2}}, \sqrt{b_{2}-x_{7}}
$$

unter gleichen Beschrānlungen der Betrachtung unterworfen werden.

\section{$\$ 2$.}

Spaltung des vorgelegten Umbehrproblems in zwei Umzehrprobleme.

Es verden jetzt 2 wei Hälfşariable $u_{1}, u_{2}$ eingeführt durch duc Gleichungen:

$$
u_{1}=\int_{a_{1}}^{x_{4}} \frac{d x_{1}}{\sqrt{\left(x_{1}-a_{2}\right)\left(b_{1}-x_{1}\right)}}, \quad u_{2}=\int_{a_{4}}^{x_{2}} \frac{d z_{2}}{\left.V_{1} x_{2}-a_{3}\right)\left(b_{1}-x_{2}\right)}
$$

Die Auflösung dieser Gleichangen giebt umgekehrt: 


$$
\left\{\begin{aligned}
x_{1} & =\frac{a_{1}+b_{1}}{2}+\frac{a_{1}-b_{1}}{2} \cos u_{1}, \\
\sqrt{\left(x_{1}-a_{1}\right)\left(b_{1}-x_{1}\right)} & =-\frac{a_{1}-b_{1}}{2} \sin u_{1}, \\
x_{2} & =\frac{a_{2}+b_{1}}{2}+\frac{a_{2}-b_{2}}{2} \cos u_{2}, \\
\sqrt{\left(x_{2}-a_{2}\right)\left(b_{2}-x_{2}\right)} & =-\frac{a_{2}-b_{2}}{2} \sin u_{2} .
\end{aligned}\right.
$$

Ferner kann man setzen:

$$
\left\{\begin{array}{l}
\sqrt{x_{1}-a_{1}}=\sqrt{b_{1}-a_{1}} \cdot \sin \frac{u_{1}}{2}, \sqrt{x_{2}-a_{2}}=\sqrt{\overline{b_{2}-a_{2}}} \cdot \sin \frac{u_{2}}{2}, \\
\sqrt{b_{1}-x_{1}}=\sqrt{\overline{b_{1}-a_{1}}} \cdot \cos \frac{u_{1}}{2}, \sqrt{b_{2}-x_{2}}=\sqrt{\overline{b_{2}-a_{2}}} \cdot \cos \frac{u_{2}}{2} .
\end{array}\right.
$$

Hiernach bleiben $x_{1}, x_{2}$ für alle positiven und negativen reellen Werthe der Variablen $u_{1}, u_{2}$ immer zwischen den durch die Ungleichungen (5) angegebenen Grenzen. Ueberdies entsprechen sich die Werthe:

$$
\left\{\begin{array}{l}
x_{1}=a_{1}: u_{1}=0, \quad x_{1}=b_{1}: u_{1}=\pi \\
x_{2}=a_{2}: u_{2}=0, \quad x_{2}=b_{2}: u_{2}=\pi
\end{array}\right.
$$

Setzt man unter Ausführung der Substitutionen (9):

$$
\begin{cases}f_{\alpha 1}\left(x_{1}\right)=p_{\alpha 1}\left(u_{1}\right), & f_{\alpha \mathrm{Q}}\left(x_{2}\right)=p_{\alpha 2}\left(u_{2}\right), \\ g_{\alpha 1}\left(x_{1}\right)=q_{\alpha 1}\left(u_{1}\right), & g_{\alpha 2}\left(x_{2}\right)=q_{\alpha 2}\left(u_{2}\right), \\ \frac{g_{\alpha 1}\left(x_{1}\right)}{V / f_{\alpha 1}\left(x_{1}\right)}=\frac{q_{\alpha 1}\left(u_{1}\right)}{\sqrt{p_{\alpha 1}\left(u_{1}\right)}}=\lambda_{\alpha_{1} 1}\left(u_{1}\right), & \frac{g_{\alpha 2}\left(x_{2}\right)}{\sqrt{f_{\alpha 22}\left(x_{2}\right)}}=\frac{q_{\alpha 2}\left(u_{2}\right)}{\sqrt{p_{\alpha 2}\left(u_{21}\right)}}=h_{\alpha \Sigma}\left(u_{2}\right),\end{cases}
$$

so sind die Functionen $p_{\alpha 1}\left(u_{1}\right), q_{\alpha 1}\left(u_{1}\right), h_{\alpha 1}\left(u_{1}\right)$ und $p_{\alpha 2}\left(u_{2}\right), q_{\alpha 2}\left(u_{2}\right)_{\text {, }}$ $h_{s 2}\left(u_{2}\right)$ der Variablen $u_{1}$, respective $u_{2}$ gerade und mit $2 \pi$ periodisch. Nach den Voraussetzungen des $\$ 1$ bleiben für alle reellen Werthe von $u_{1}, u_{2}$ die Funetionen $p_{\alpha 1}\left(u_{1}\right)$ und $p_{\alpha 2}\left(u_{2}\right)$ positiv und von 0 und $\infty$ verschieden, und behalten die Functionen $q_{\alpha 1}\left(u_{1}\right)$ und $q_{\alpha 2}\left(u_{2}\right)$, ohne $\infty$ zu werden, immer ihr Vorzeichen. Daher werden $h_{\alpha 1}\left(u_{1}\right)$ und $h_{a 2}\left(u_{2}\right)$ für alie reellen Werthe von $u_{1}, u_{2}$ endlich sein und niemals ihr Vorzeichen wechseln.

Zugleich verwandelt sich die Determinante:

$$
D\left(x_{1}, x_{2}\right)=h_{11}\left(u_{1}\right) \cdot h_{2 z}\left(u_{2}\right)-h_{21}\left(u_{1}\right) \cdot h_{12}\left(u_{2}\right)=h\left(u_{1}, u_{2}\right)
$$

in eine gerade Function der beiden Variablen $u_{1}, u_{2}$, welche für alle reellen. Werthe von $u_{1}, u_{2}$ ein festes Vorzeichen bat, für solche Werthe $u_{1}, u_{2}$ niemals verschwindet ond in Bezug auf jede der Variablen $u_{1}, u_{2}$ mit $2 \pi$ periodisch ist.

Die Function $E\left(x_{1}, x_{2}\right)$ geht nach Substitution der Werthe (9) in eine für alle reellen Werthe $u_{1}, u_{2}$ eindeutige ond endliche Function der Variabeln $u_{1}, u_{2}$ über: 


$$
E\left(x_{1}, x_{2}\right)=H\left(u_{1}, u_{2}\right),
$$

die gerade ist und in Bezug auf jedes der Argamente $u_{1}, u_{2}$ die Periode $2 \pi$ hat. Fine eindeutige Function der vier Wurzelgrössen (7) würde mit Rücksicht auf die Gleichungen (10) als eine eindeutige, im Allgemeinen nicht mehr gerade Fuuction der Variablen $u_{1}, u_{2}$ nit der Periode $4 \pi$ in Bezug auf jede derselben sich darstellen.

Zwischen $u_{1}, u_{2}$ und $t_{1}, t_{2}$ bestehen jelat die Relationen:

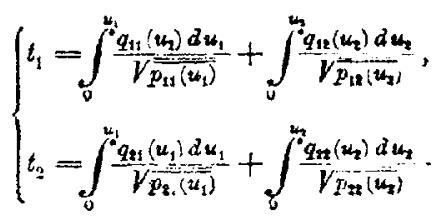

Da nun, wie in $\$ \S 3 ; 4$ weiter zu erörtern, für alle reellen Werthe von $t_{1}, t_{2}$ auch $u_{1}, u_{2}$ reelle Werthe annehmen, so bleiben $x_{1}, x_{2}$ auch für alle reellen Werthe der Variablen $t_{1}, t_{2}$ zwischen den onier (5) angegebenen Grenzen.

Nachdem so von dem Umkehrproblem (1) das trigonometrische Umbehrproblem in (8) abgespalten ist, bleibt das Umbehrproblem (15) übrig, welches vor dem ursprünglich vorgelegten sich dadurch auszeichnet, dass die Quadratwurzein in (15) ihr Vorzeichen nicht mebr wechseln kōnnen.

\section{ร 3.}

Die Eindentigkeit der Functionen $t_{1}, t_{2}$ von $u_{1}, u_{z}$.

Die Functionen $t_{1}, t_{2}$ sind von $u_{1}, u_{t}$ abhägig gemacht durch die Gleichungen:

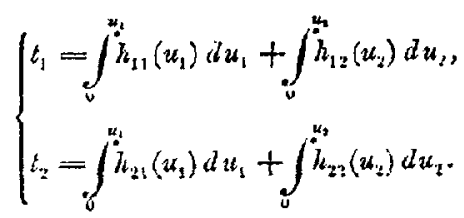

Es werde zur Abhïroung gesetzt:

$$
\begin{cases}\psi_{11}\left(u_{1}\right)=\int_{0}^{u_{4}} h_{11}\left(u_{1}\right) d u_{1}, & \psi_{12}\left(u_{2}\right)=\int_{0}^{u_{2}} \vec{h}_{12}\left(u_{2}\right) d u_{2}, \\ \psi_{21}\left(u_{1}\right)=\int_{0}^{u_{1}} h_{21}\left(u_{1}\right) d u_{1}, & \psi_{22}\left(u_{2}\right)=\int_{0}^{u} h_{22}\left(u_{2}\right) d u_{2},\end{cases}
$$


sodass:

$$
\left\{\begin{array}{l}
t_{1}=\psi_{1}\left(u_{1}, u_{2}\right)=\psi_{11}\left(u_{1}\right)+\psi_{12}\left(u_{2}\right), \\
t_{2}=\psi_{2}\left(u_{1}, u_{2}\right)=\psi_{21}\left(u_{1}\right)+\psi_{22}\left(u_{2}\right) .
\end{array}\right.
$$

Umgetehrt sei:

$$
u_{1}=\varphi_{1}\left(t_{1}, t_{2}\right), \quad u_{2}=\varphi_{2}\left(t_{1}, t_{2}\right) .
$$

Jedes der Integrale $\psi_{\alpha 1}\left(u_{1}\right)$ and $\psi_{\alpha 2}\left(u_{2}\right)$ hat für jeden reellen Werth von $u_{1}$, respective $u_{2}$ selbst einen bestimmten, mit dem Argument stetig sich ändernden Werth, da die Functionen $h_{a 1}\left(u_{1}\right)$ und $h_{\alpha 2}\left(u_{2}\right)$ für alle reellen Werthe von $u_{1}$, respective $u_{y}$ eindentig, endlich und stetig sind und besondere Singularitäten derselben ausgeschlossen werden. Da überdies die Functionen $h_{\alpha 1}\left(u_{1}\right)$ und $h_{\alpha 2}\left(u_{2}\right)$ gerade Functionen sind, so ist:

$$
\psi_{\alpha 1}\left(-u_{1}\right)=-\psi_{\alpha_{1}}\left(u_{1}\right), \quad \psi_{\alpha 2}\left(-u_{2}\right)=-\psi_{\alpha 2}\left(u_{2}\right)
$$

und somit auch:

$$
\left\{\begin{array}{l}
\psi_{1}\left(-u_{1},-u_{2}\right)=-\psi_{1}\left(u_{1}, u_{2}\right) \\
\psi_{2}\left(-u_{1},-u_{2}\right)=-\psi_{2}\left(u_{1}, u_{2}\right)
\end{array}\right.
$$

Es folgt also zunächst:

Die Functionen $t_{\mathrm{t}}=\psi_{1}\left(u_{1}, u_{2}\right)$ und $t_{2}=\psi_{2}\left(u_{1}, u_{2}\right)$ sind für all reellen $W$ erthepacre $u_{1}, u_{2}$ eindeutige und stetige, sowie ungerade $F u n c$. tionen von $u_{1}, u_{2}$.

Man denke sich die Functionen $t_{1}$ und $t_{2}$ von $u_{1}, u_{2}$ geometrisel dargestellt, indem man $t_{1}$ und $t_{2}$ als Ordinaten auf einer Abscissen. ebene $u_{1}, u_{2}$ aufträgt. Es werden dann zwei Flächen entstehen, welch für jeden Punkt $u_{1}, u_{2}$ der Abscissenebene eine und nur eine Ordinat besitzen. Aus der Differentialgleichung der Fläche $t_{\alpha}=\psi_{\alpha}\left(u_{1}, u_{2}\right)$ :

$$
d t_{\alpha}=h_{\alpha 1}\left(u_{1}\right) d u_{1}+h_{c 2}\left(u_{2}\right) d u_{2}
$$

geht noch hervor, dass die Fläche in jedem ibrer Punkte eine bestimmte ihre Normalenrichtung stetig verändernde Tangentialebene hat, und das diese letztere niemals der Abscissenebene $u_{1}, u_{2}$ parallel werden kann da $h_{\alpha 1}\left(u_{1}\right)$ und $h_{\alpha 2}\left(u_{2}\right)$, bei der über die Determinante $D\left(x_{1}, x_{2}\right)=h\left(u_{1}, u_{2}\right.$ in $\$ 1$ gemachten Voraussetzung, niemals gleichzeitig versehwinden.

Die Fläche $t_{\alpha}=\psi_{\alpha}\left(u_{1}, u_{2}\right)$ sei im Besonderen längs der Geraden

$$
u_{2}=\varepsilon_{\alpha} u_{1}+a
$$

der Abscissenebene betrachtet, wo $\varepsilon_{\alpha}= \pm 1$ und $a$ eine beliebig Constante ist. Man hat für die Punkte djeser Geraden:

$$
\begin{aligned}
t_{\alpha} & =\int_{j}^{u_{2}} h_{\alpha 1}\left(u_{1}\right) d u_{1}+\varepsilon_{\alpha} \int_{j}^{u_{2}} h_{\alpha 2}\left\{\varepsilon_{\alpha} u_{1}+a\right) d u_{1}+\int_{j}^{a} h_{\alpha 2}\left(u_{2}\right) d u_{2} \\
& =\int_{\delta}^{u_{x}}\left(h_{\alpha 1}\left(u_{1}\right)+\varepsilon_{\alpha} h_{\alpha 2}\left(\varepsilon_{\alpha} u_{1}+a\right)\right) d u_{1}+A,
\end{aligned}
$$


wo:

$$
A=\int_{0}^{A} h_{\alpha \Omega}\left(u_{2}\right) d t_{y} .
$$

Da nun $h_{\alpha 1}\left(u_{1}\right)$ und $h_{\alpha \pm}\left(u_{2}\right)$ für alle reellen Werthe ron $u_{1}$ und $u_{2}$ je das nämliche Vorzeichen besitzen und niemals gleichzeitig verschwinden, so kann man $\varepsilon_{a}= \pm 1$ stets so wäblen, dass die Function $h_{\alpha 1}\left(u_{1}\right)+\varepsilon_{\alpha} h_{\alpha 2}\left(\varepsilon_{\alpha} u_{1}+a\right)$ für alle reellen Werthe ron $u_{1}$ beständig positiv oder beständjg negativ und niemals gleich 0 ist. Hiernach wird $t_{a}$ längs der betrachteten Geraden bestāndig wachsen adex beständig abnehmen, während $u_{1}$ stetig wachsend vou $-\infty$ bis $+\infty$ läuft.

Dabei wird aber $t_{\alpha}$ längs der Geraden nach beiden Seiten derselbeu hin auch schliesslich $-\infty$ und respective $+\infty$ werden, da dio Function $h_{\alpha 1}\left(u_{1}\right)+\varepsilon_{\alpha} h_{\alpha q}\left(\varepsilon_{\alpha} u_{1}+a\right)$ eine periodische Function von $u_{1}$ mit der Periode $2 \pi$ und $A$ eine für jedes endliche $a$ eadliche Constante ist.

Es giebt also stets ein System paralleler gerader Linien in der Abscissenebene $u_{1}, u_{2}$, lïngs deren jeder in der einen adcr axideren Richtung die Ordinate der Flüclve $t_{1}=\psi_{1}\left(u_{1}, u_{2}\right)$ oder $t_{2}-\psi_{2}\left(u_{1}, u_{2}\right)$ stetig voachsend alle Werthe von $-\infty$ bis $+\infty$ durchläuft.

Verbindet man alle Punlte $u_{1}, u_{2}$ der Abscissenebene, denen gleiche Ordinaten $t_{1}$ oder $t_{2}$ entsprechen, so erhält man beztiglich Curven $t_{1}=$ const. und $t_{2}=$ const., welche die Orthogonalprojectionen der Höhenlinien der Flächen $t_{1}=\psi_{1}\left(u_{1}, u_{2}\right)$ und $t_{2}=\psi_{2}\left(u_{1}, u_{2}\right)$ sind. Die Differentialgleichang einer Curve $t_{\alpha}=$ const. ist:

$$
0=h_{\alpha 1}\left(u_{1}\right) d u_{1}+h_{\alpha q}\left(u_{2}\right) d u_{2} \text {, }
$$

woraus mit Rücksicht darauf, dass $h_{a}\left(u_{1}\right)$ und $h_{\alpha z}\left(u_{2}\right)$ für alle reellen Werthe $u_{1}, u_{2}$ eindeutig, endlich und stetig und niemals beide 0 sind, hervorgeht, dass die Curve in jedem ihrer Punkte eine einzige bestimmte Tangente hat. Es folgt daher:

Jede Curve $t_{1}=$ const. und $t_{2}=$ const. Inllet cinen einzigen, un. verzweigten vom Unendichen her ins Unendliche auriiclilauf(nden Zug ${ }^{*}$ ).

* Die Gesammtheit aller Pankte der Ebene $u_{1}$, $u_{k}$. deren Coordinatea ihrom alsoluten Betrage nach eine beliebig grosse eudliche Grenze nicht überachreiten, bildet ein einfach znsammenhängendes Flächenstïck; nach dem rorliogenden Satze lizuft jede Corve $t_{\alpha}$ const. als einfacher, wich walbut nicht whneidender Curvenzug von einem Rardpunkte dieses Flabenutickes 20 einem anderen Inadpunkte. Der vorliegende und folgende Satz entoprechen daher einer der Bedingangen für die eindeutige Unkehrnog eines Fonctionenastems, welche Lipuchitz, Beitrïge zu der Theorie der Umkebrang eine Funetioncnuyutems, Nachrichten dar bgl. Ges. d. Wiscenschaften zh Gottingen, 1870, S, 439, angiebt 
Die Verbindung der beiden letzten Sätze giebt weiter:

Die Funetion $t_{1}=\psi_{1}\left(u_{1}, u_{2}\right)$, und ebenso $t_{2}=\psi_{2}\left(u_{1}, u_{2}\right)$, nimmt joden Werth zacischen $-\infty$ und $+\infty$ längs einer und nut einer vom Unendlichen her ins Unendliche zurïchlaufenden, unverzveigten Curve der Abscisseneluene an.

\section{$\$ 4$.}

Die Eindeutigkeit der Functionen $u_{1}, u_{2}$ von $t_{1}, t_{2}$.

Die Tangenten zweier Curven $t_{1}=$ const. und $t_{2}=$ const. in einem Schnittpunkte $u_{1}, u_{2}$ derselben sind in laufenden Coordinaten $u_{1}^{\prime}, u_{2}^{\prime}$ :

$$
\begin{aligned}
& h_{11}\left(u_{1}\right) \cdot\left(u_{1}^{\prime}-u_{1}\right)+h_{12}\left(u_{2}\right) \cdot\left(u_{2}^{\prime}-u_{2}\right)=0, \\
& h_{21}\left(u_{1}\right) \cdot\left(u_{1}^{\prime}-u_{1}\right)+h_{22}\left(u_{2}\right) \cdot\left(u_{2}^{\prime}-u_{2}\right)=0 ;
\end{aligned}
$$

diese Tangenten können niemals zusammenfallen, da die Determinante $h\left(u_{1}, u_{2}\right)$ dieser beiden linearen Gleichnngen niemals verschwindet. Mit andern Worten:

Zwei Curven $t_{1}=$ const. und $t_{2}=$ const. Können sich niemals unter dem Winltel 0 oder $\pi$ schneiden.

Man denke sich nun jede solche Gurve mit einem bestimmten Durchlaufungssinne versehen, der sich von Curve zu Curve eines jeden der beiden Systeme $t_{1}=$ const. und $t_{2}=$ const. continuirlich übertrage. Man betrachte ferner, indem man längs einer Curve $t_{1}=$ const. in ihrein Durehlaufungssinne fortschreitet, die Aenderung in der Richtung der sie schneidenden Curven $t_{2}=$ const. Diese Richtung wird sich ron Punkt zu Punkt der ersteren Carve stetig ändern, obne je mit der Richtung derselben zusammenzufallen oder ihr gerade entgegengesetzt zu sein. Daher durchschneiden, mit Bezug auf den festgesetzten Durchlaufungssinn, die Curven $t_{2}=$ const. die Curve $t_{1}=$ const. immer von derselben Seite her, ron der rechten oder von der linken. Es ist somit ausgeschlossen, dass eine Curve $t_{2}=$ const. mehr als einmal die Curre $t_{1}=$ const. schneidet.

Eine Curve $t_{1}=$ const. und eine Curve $t_{2}=$ const. in der Abscissen-

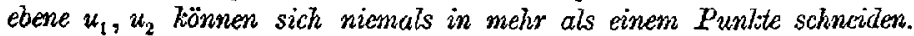

Denkt man sich jetat eine Ordinate $t_{1}$ der Fiäche $t_{1}=\psi_{1}\left(u_{1}, u_{2}\right)$ und eine Ordinate $t_{2}$ der Fläche $t_{2}=\psi_{2}\left(u_{1}, u_{2}\right)$ gegeben, so kann es nicht mehr als einen Punkt $u_{1}, u_{2}$ der Abscissevebene geben, wo gleichzeitig die erstere Fläche die Ordinate $t_{1}$ und die letztere die Ordinate $t$. besitzt.

Es gehört also zu eincm reellen Werthepaare $t_{1}, t_{2}$, nichit meinr als cin Werth jeder der beiden Functionen $u_{1}=\varphi_{1}\left(t_{1}, t_{2}\right)$ und $u_{2}=\varphi_{2}\left(t_{1}, t_{2}\right)$. Die diesen Fuuctionen entsprechendeu Flächen mögen mit den 
verticalen Ordinaten $u_{1}, t_{2}$ iber einer horizontalen Abscissenebene $t_{1}, t_{2}$ construirt werden. Die Differentialgleichnongen dieser beiden Füchen lisuten:

$$
\begin{aligned}
& d u_{1}=\frac{h_{n}\left(u_{1}\right)}{h\left(u_{1}, u_{2}\right)} d t_{1}-\frac{h_{1}\left(u_{2}\right)}{h\left(\omega_{1}, u_{2}\right)} d t_{2}, \\
& d u_{2}=-\frac{h_{21}\left(u_{1}\right)}{h\left(u_{1}, u_{2}\right)} d t_{1}+\frac{h_{11}\left(\omega_{1}\right)}{h_{1}\left(w_{1}, u_{2}\right)} d t,
\end{aligned}
$$

wo für die Coefficienten die Integrabilitätsbedingungen erfullt sind. Geht man nun von einem beliebig gegebenen Werthepaare $u_{1}, u_{2}$ ans, so gehört zu diesem nach $\$ 3$ ein und nur ein Werthepaar $t_{1}, t_{2}$ and man kann je einen Punkt der beiden Flächen $u_{3}=\varphi_{1}\left(t_{1}, t_{2}\right)$ und $u_{2}=\varphi_{2}\left(t_{1}, t_{2}\right)$ construiren. Die Tangentialebenen in diesen Punkten sind in laufenden Coordinaten $u_{1}^{\prime}, t_{1}^{\prime}, t_{2}^{\prime}$, respective $u_{2}^{\prime}, t_{1}^{\prime}, t_{1}^{\prime}$ :

$$
\begin{aligned}
& u_{1}^{\prime}-u_{1}=\frac{h_{2}\left(u_{2}\right)}{h\left(u_{1}, u_{2}\right)}\left(t_{1}^{\prime}-t_{1}\right)-\frac{h_{12}\left(u_{2}\right)}{h\left(u_{1}, u_{1}\right)}\left(t_{2}^{\prime}-t_{2}\right), \\
& u_{2}^{\prime}-u_{2}=-\frac{h_{21}\left(u_{1}\right)}{h\left(u_{1}, u_{2}\right)}\left(t_{1}^{\prime}-t_{1}\right)+-\frac{h_{11}\left(u_{1}\right)}{h\left(u_{1}, u_{2}\right)}\left(t_{2}^{\prime}-t_{2}\right) .
\end{aligned}
$$

Geht man jetzt von $t_{1}, t_{2}$ zu einem beliebigen, unendlich nahen Nachbarpunkte $t_{1}, t_{2}$ der Abscissenebene $t_{1}, t_{2}$ über, so geben diese Gleichungen die zugehörigen Werthe ron $u_{1}^{*}, u_{2}^{\prime}$; fur die entsprechenden Punlito der Flächen $u_{1}=\varphi_{1}\left(t_{1}, t_{2}\right)$ und $u_{1}=\varphi_{2}\left(t_{1}, t_{2}\right)$ kann man dann wieder die Gleichnngen der Tangentialebene angeben und so fortfabrend die Flächen weiter und weiter construiren. Soweit diese Fortsekzung auch geführt wird, sie bleibt immer eindeutig und bestimmt. Nimmt man hierzu noch, dass zu einem Werthepare $t_{1}, t_{2}$ nie mehr als ein Werth von $u_{1}$ oder $u_{2}$ gehört, so folgt, dass jede der beiden Flächen $u_{1}-\varphi_{1}\left(t_{1}, t_{2}\right)$ und $u_{2}=\varphi_{2}\left(t_{1}, t_{2}\right)$ die Abscissenebene $t_{1}, t_{2}$ in ihrer ganzen Ausdebnung einfach über- respective nnter-decken muss. Die Zusammenfassung dieser Schlüsse fübrt aber zu dem Satze:

Die Functionen $u_{1}=\varphi_{1}\left(t_{1}, t_{2}\right)$ und $u_{2}-\varphi_{2}\left(t_{1}, t_{3}\right)$ sind für alle reellen Werthepaarc $t_{1}, t_{2}$ eindetatige und stetige Functionen von $t_{1}, t_{2}$.

$\S 5$.

Dio doppelte reelle Periodicität dor Ponctionen von $t_{1}, t_{z}$.

Nachdem die Eindeutigkeit der Functionen

$$
u_{1}=\varphi_{1}\left(t_{1}, t_{2}\right), \quad u_{2} \varphi_{2}\left(t_{1}, t_{2}\right)
$$

bewiesen ist, kann man sofort eive weitere charakteristische Eigenschaft derselben herleiten. $\mathrm{Da}_{\mathrm{a}}$ dämlich die functionen $h_{\mathrm{a}}\left(u_{1}\right)$ und $h_{a 2}\left(u_{2}\right)$ nach \& 2 doppelt rell perjodisch sind mit den zusammengehörigen Periodicitätsmodaln $2 \pi, 0$ und $0,2 \pi$, nand mit Rüclsicht anf die Gleichangen (15) and (17): 


$$
\frac{\partial \psi_{\alpha}\left(u_{1}, u_{2}\right)}{\partial u_{1}}=h_{\alpha 1}\left(u_{1}\right), \quad \frac{\partial \psi_{\alpha}\left(u_{1}, u_{2}\right)}{\partial u_{2}}=\hbar_{\alpha 2}\left(u_{2}\right),
$$

so folgt sofort:

$$
\frac{\partial \psi_{\alpha}\left(u_{1}+2 \pi, u_{2}+0\right)}{\partial u_{1}}=\frac{\partial \psi_{\alpha}\left(u_{1}, u_{2}\right)}{\hat{\partial} u_{1}}, \frac{\partial \psi_{\alpha}\left(u_{1}+2 \pi, u_{2}+0\right)}{\partial u_{2}}=\frac{\partial \psi_{\alpha}-\frac{\left(u_{1}, u_{2}\right)}{\hat{\sigma} u_{2}}}{,}
$$

und entsprechend mit Bezug auf die Aenderung ron $u_{1}, u_{2}$ um $0,2 \pi$. Multiplicirt man diese beiden Gleichungen bezüglich mit $d u_{1}, d u_{2}$ und addirt sie, so ergiebt sich für das vollständige Differential:

$$
d \psi_{\alpha}\left(u_{1}+2 \pi, u_{2}+0\right)=d \psi_{\alpha}\left(u_{1}, u_{2}\right) \text {. }
$$

Durch Integration erbält man hieraus, unter $\omega_{\alpha 1}$ eine Constante verstehend:

$$
\psi_{\alpha}\left(u_{1}+2 \pi, u_{2}+0\right)=\psi_{\alpha}\left(u_{1}, u_{2}\right)+2 \omega_{\alpha \lambda}
$$

und auf demselben Wege:

$$
\psi_{c}\left(u_{1}+0, u_{2}+2 \pi\right)=\psi_{c}\left(u_{1}, u_{2}\right)+2 \omega_{\alpha 2} .
$$

Wegen der wechselseitigen Eindeutigkeit der Beziehungen zwischen den Variablen $u_{1}, u_{2}$ einerseits und $t_{1}, t_{2}$ andererseits schliesst man hieraus weiter:

$$
\left\{\begin{array}{l}
u_{1}+2 \pi=\varphi_{1}\left(t_{1}+2 \omega_{11}, t_{2}+2 \omega_{21}\right) \\
u_{2}+0=\varphi_{2}\left(t_{1}+2 \omega_{11}, t_{2}+2 \omega_{21}\right) \\
u_{1}+0=\varphi_{1}\left(t_{1}+2 \omega_{12}, t_{2}+2 \omega_{22}\right) \\
u_{2}+2 \pi=\varphi_{2}\left(t_{1}+2 \omega_{12}, t_{2}+2 \omega_{22}\right) .
\end{array}\right.
$$

So oft die Variablen $t_{1}, t_{2}$ respective um $2 \omega_{11}, 2 \omega_{21}$ oder $2 \omega_{12}, 2 \omega_{22}$ vochsen, nehmen $u_{1}, u_{2}$ respective un $2 \pi, 0$ oder $0,2 \pi z u$.

Da aber die Function $H\left(u_{1}, u_{2}\right)$ in (14) in Bezug auf $u_{1}, u_{2}$ die Periodicitätsmoduln $2 \pi, 0$ und $0,2 \pi$ hat, so gelangt man zu dem Resultate, dass diese Function, als Function $G\left(t_{1}, t_{4}\right)$ von $t_{1}, t_{2}$ betrachtet, doppelt periodisch ist mit den Periodenpaaren $2 \omega_{11}, 2 \omega_{21}$ und $2 \omega_{12}, 2 \omega_{22}$.

Um die Werthe der Constanten $\omega_{\alpha \beta} z u$ bestimmen, entnimmt man aus den Gleichungen (20) mit $u_{1}=-\pi, u_{2}=0$ :

$$
\psi_{\alpha}(\pi, 0)=\psi_{\alpha}(-\pi, 0)+2 \omega_{\alpha 1}
$$

und hieraus mit Rücksicht auf (19):

ebenso erhält man:

$$
2 \psi_{\alpha}(x, 0)=2 \omega_{a l}
$$

$$
2 \psi_{\alpha}(0, \pi)=2 \omega_{\alpha} \text {. }
$$

Da aber den Wertheparen $\pi, 0$ und $0, \pi$ von $u_{1}, u_{2}$ nach (11) die Werthepaare $b_{1}, a_{2}$ und $a_{1}, b_{2}$, pon $x_{1}, x_{2}$ entsprechen, so ist: 


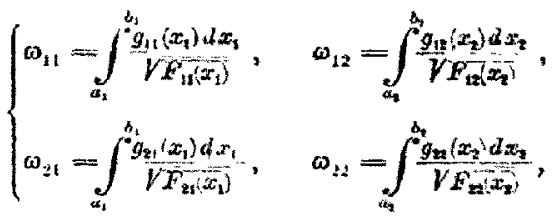

wo die Quadratworzeln sämmtlich positiv genommen werden können.

Das Gesammtresultat der vorstehenden Entwichlungen kann sonach folgendermaassen formulirt werden:

Eine belialig gegebene Function $E\left(x_{1}, x_{2}\right)$ der aberen Grenean $x_{1}, x_{2}$ der Integralsummen.

$$
\begin{aligned}
& t_{1}=\int_{a_{1}}^{x_{2}} \frac{g_{11}\left(x_{1}\right) d x_{4}}{\sqrt{F_{\mathrm{t}}\left(x_{1}\right)}}+\int_{a_{2}}^{x_{2}} \frac{s_{21}\left(x_{2}\right) d x_{2}}{\sqrt{k_{12}\left(x_{2}\right)}}, \\
& t_{2}=\int_{a_{1}}^{x_{1}} \frac{g_{21}\left(x_{1}\right) d x_{1}}{\sqrt{F_{21}^{\prime}\left(x_{1}\right)}}+\int_{a_{1}}^{x_{2}} \frac{g_{z}\left(x_{2}\right) d x_{2}}{\sqrt{F_{12}}},
\end{aligned}
$$

welche für alle den Ongleichungen $a_{1}<x_{1}<b_{1}, a_{2}<x_{2}<b_{1}$ gonügon. den Werthepaare $x_{1,} x_{2}$ eindeutig, endlich und stetig ist, wird unter den in $\$ 1$ angegeberen Voraussetzanzen cins für alle reellen Werthepaarc von $t_{1}, t_{2}$ eindeutige, endliche und stetige $F$ unction $G\left(t_{1}, t_{1}\right)$ von $t_{1}, t_{2}$, die ïherdies gerade und doppelt periodisch mit den Periadenpaaren $2 \omega_{11}, 2 \omega_{31}$ und $2 \omega_{12}, 2 \omega_{22}$ ist.

In derselben Beziehung zu $t_{1}, t_{2}$ stehen entsprechende eindeutige Functionen der Variablen $x_{1}$ und $x_{2}$ und der Wurzelgrössen:

$$
\sqrt{x_{1}-a_{1}}, \sqrt{b_{1}-x_{1}}, \sqrt{x_{2}-a_{2}}, \sqrt{b_{2}-x_{2}} \text {, }
$$

nur dass diese im Allgemeinen nicht mekr gerade sind und dass sie die Periodenparare $4 \omega_{11}, 4 \omega_{31}$ und $4 \omega_{12}, 4 \omega_{32}$ besitzen.

\section{$\$ 6$.}

Die Omkehrung der hyperelliptischen fntegrale im reellen Gobiot.

Das behandelte Umkehrproblem ist cine aul das reelle Gebiet beschränkte Verallgemeinerung des Jacobi'schen Unkehrproblems der hyperelliptischen Integrale 1. Gattong rom Geschlecht $p=2$ :

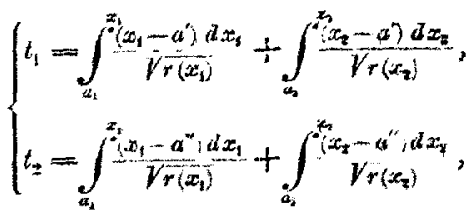


worin:

$$
\begin{gathered}
r(x)=\left(a_{0}-x\right)\left(a_{1}-x\right)\left(a_{2}-x\right)\left(a_{3}-x\right)\left(a_{4}-x\right), \\
a_{0}<a_{1}<a_{2}<a_{3}<a_{4},
\end{gathered}
$$

und $a^{\prime}, a^{\prime \prime}$ irgend 2 von den Verzweigungspunkten $a_{0}, a_{1}, a_{2}, a_{3}, a_{1}$ der Quadratwurzel $\sqrt{r(x)}$ sind. Es ist in diesem Falle nach den Bezeichnungen des $\S 1$ :

$$
\begin{gathered}
g_{11}(x)=g_{12}(x)=x-a^{\prime}, \quad g_{21}(x)=g_{22}(x)=x-a^{\prime \prime}, \\
F_{11}(x)=F_{12}(x)=F_{21}(x)=F_{22}(x)=r(x), \\
f_{11}(x)=f_{21}(x)=\left(x-a_{0}\right)\left(a_{3}-x\right)\left(a_{4}-x\right), \\
f_{12}(x)=f_{22}(x)=\left(x-a_{0}\right)\left(x-a_{1}\right)\left(x-a_{2}\right), \\
D\left(x_{1}, x_{2}\right)=\frac{\left(x_{2}-x_{1}\right)\left(a^{\prime \prime}-a^{\prime}\right)}{\gamma / \bar{f}_{11}\left(x_{1}\right)} \sqrt{f_{22}\left(x_{2}\right)}
\end{gathered}
$$

Zugleich sind die Bezeichnongen $a_{1}, a_{2}, a_{3}, a_{4}$ an Stelle von $a_{1}, b_{1}$, $a_{2}, b_{2}$ getreten. Die Bedingungen des $\$ 1$ sind, wie man sofort sieht, alle erfullt, sobald $a^{\prime \prime}-a^{\prime}=\mid=0$ ist. Es sind also z. B. die Functionen:

$$
\begin{gathered}
\sqrt{\sqrt{x_{1}-a_{0}} \sqrt{x_{2}-a_{0}}}, \sqrt{x_{1}-a_{1}} \sqrt{x_{2}-a_{1}}, \sqrt{a_{2}-x_{1}} \sqrt{x_{2}-a_{2}}, \\
\sqrt{a_{3}-x_{1}} \sqrt{x_{2}-a_{3}}, \sqrt{\overline{a_{1}-x_{1}}} \sqrt{a_{4}-x_{2}}
\end{gathered}
$$

als in den Intervallen $a_{1}<x_{1}<a_{3}, a_{3}<x_{2}<a_{4}$ eindeutige, endliche und stetige Functionen ron

$$
x_{1}, x_{2}, \sqrt{x_{1}-a_{3}}, \sqrt{a_{2}-x_{1}}, \sqrt{x_{2}-a_{3}}, \sqrt{a_{4}-x_{2}}
$$

nach $\$ 5$ eindeutige, endliche und stetige Functionen von $t_{1}, t_{2}$ mit 2 Paaren reeller Perioden.

Die vorstehend entwickelte Theorie giebt aber im reellen Gebiete auch die Lösung des Umkehrproblems (23), wenn die Integrale derselben nicht mehr von der 1. Gattung, sondern etwa theilweise von der 2. Gattung sind, z. B.

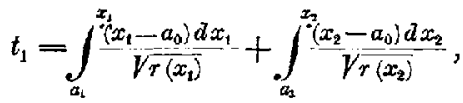

$$
\begin{aligned}
& t_{2}=\int_{a_{2}}^{x_{1}} \frac{\left(x_{1}-a_{0}\right)\left(x_{1}-a_{2}\right) d x_{1}}{\sqrt{r\left(x_{1}\right)}}+\int_{a_{2}}^{x_{2}} \frac{\left(x_{2}-a_{4}\right)\left(x_{2}-a_{2}\right) d x_{2}}{\sqrt{r\left(x_{2}\right)}} .
\end{aligned}
$$

Auch hier sind alle Voraussetzungen des $\$ 1$ erfüllt. Dies gilt aber auch dann noch, wenn in (23) $r(x)$ ron höherem als dem 6. Grade ist und in $x=a_{1}, a_{2}$ und $x=a_{3}, a_{4}$ je zwei nebeneinanderfolgende Nullpunkte hat, sodass die Integrale hyperelliptiseh von höherem Geschlecht als $p=2$ werden. 
$\$ 7$.

Die Darstallung der botrachtaten doppelt reoll periodischan Functionen. Die Function $E\left(x_{1}, x_{2}\right)=G\left(t_{1}, t_{2}\right)$ in $\$ 5$ hat die Eigenschaften:

$$
\left\{\begin{aligned}
G\left(-t_{1},-t_{2}\right) & =G\left(t_{1}, t_{2}\right), \\
G\left(t_{1}+2 \omega_{13}, t_{2}+2 \omega_{21}\right) & =G\left(t_{1}, t_{2}\right), \\
G\left(t_{1}+2 \omega_{12}, t_{2}+2 \omega_{22}\right) & =G\left(t_{1}, t_{3}\right) .
\end{aligned}\right.
$$

Führt man nun statt $t_{1}, t_{2}$ 2wei neue Variable $\tau_{1}, \tau_{2}$ ein durch die Gleichungen:

$$
\tau_{1}=\frac{t_{3} \omega_{22}-t_{3} \omega_{12}}{\omega_{11} \omega_{22}-\omega_{21} \omega_{12}} x, \quad \tau_{2}=\frac{\omega_{11} t_{2}-\omega_{12} t_{1}}{\omega_{11} \omega_{22}-\omega_{11} \omega_{13}} \pi,
$$

พ०

$$
\omega=\omega_{11} \omega_{22}-\omega_{21} \omega_{12}=\int_{u_{4}}^{b_{2}} \int_{a_{2}}^{b_{2}} \frac{D\left(x_{1}, x_{1}\right) d x_{1} d x_{1}}{V\left(x_{1}-a_{1}\right)\left(b_{1}-x_{1} V\left(x_{2}-a_{2}\right)\left(x_{2}-x_{2}\right)\right.}
$$

von Null verschieden and von gleichem Vorzejchen mit $D\left(x_{1}, x_{2}\right)$ ist, setzt man ferner:

$$
G\left(t_{1}, t_{2}\right)=\Gamma\left(\tau_{1}, x_{2}\right),
$$

so sind die entsprechenden Eigenschaften der Function $\Gamma\left(\boldsymbol{r}_{1}, \boldsymbol{\tau}_{2}\right)$ in den Formeln enthalten:

$$
\left\{\begin{array}{l}
\Gamma\left(-\tau_{1},-\tau_{2}\right)=\Gamma\left(\tau_{1}, \tau_{2}\right) \\
\Gamma\left(\tau_{1}+2 \pi, \tau_{2}\right)=\Gamma\left(\tau_{1}, \tau_{2}\right) \\
\Gamma\left(\tau_{1}, \tau_{2}+2 \pi\right)=\Gamma\left(\tau_{1}, \tau_{2}\right)
\end{array}\right.
$$

Die Function $\Gamma\left(\tau_{1}, \tau_{2}\right)$ ist also eine für alle reellen Werthe von $\tau_{1}, \tau_{2}$ eindeutige, endliche und stetige Function der beiden Argumente $\tau_{1}, \tau_{2}$ und mit Bezug anf jedes der beiden Argumente periodisch mit der Periode $2 \pi$. Sie ham daher ${ }^{*}$ ) durch eine für alle Werthe von $\tau_{1}, \tau_{2}$ gleichmässig convergente, zweifach nnendliche trigonometrische Beihe dargestelst werden, welche die form hat:

$$
\begin{aligned}
& \Gamma\left(z_{1}, \tau_{2}\right)={ }_{4}^{1} A_{00}+\frac{1}{2} \sum_{1}^{\infty}\left(A_{n_{2}, 0} \cos n_{1} z_{1}+B_{n_{0}, 0} \sin n_{1} \tau_{1}\right) \\
& +\frac{1}{3} \sum_{1}^{\infty} m_{2}\left(A_{0 n_{2}} \cos n_{2} \tau_{2}+C_{0 m} \sin n_{2} z_{2}\right) \\
& +\sum_{i}^{\infty} \sum_{1}^{\infty} \sum_{x_{1}}^{\infty}\left(A_{n_{1} u_{2}} \cos n_{1} \tau_{1} \cdot \cos n_{1} r_{2}+B_{u_{1} n_{1}} \sin n_{1} \tau_{1} \cdot \cos n_{2} \tau_{2}\right. \\
& \left.+C_{n_{1} n_{2}} \cos n_{1} \tau_{1} \cdot \sin n_{2} \tau_{2}+D_{n_{1} m} \sin n_{1} \tau_{1} \cdot \sin n_{2} \tau_{2}\right)
\end{aligned}
$$

*) Vgl. Ascoli, Sulla rappresentibiliti di una funzione a doe pariabili jer serie doppia trigonometrica ond Sulle serie trigonometriche a due varinbili, Atli 
und die Fourier'sche Reihe zweier Veründerlicher ist, sodass ihre Coefficienten die Werthe haben:

$$
\begin{aligned}
& A_{\pi_{1} n_{2}}=\frac{1}{\pi^{2}} \int_{0}^{2 \pi} \int_{0}^{2 \pi} \Gamma\left(\tau_{1}, \tau_{2}\right) \cdot \cos n_{1} \tau_{1} \cdot \cos n_{2} \tau_{2} \cdot d \tau_{1} d \tau_{2}, \\
& B_{n_{2} n_{2}}=\frac{1}{\pi^{2}} \int_{0}^{2 \pi} \int_{0}^{\pi \pi} \Gamma\left(\tau_{1}, \tau_{2}\right) \cdot \sin n_{1} \tau_{1} \cdot \cos n_{2} \tau_{2} \cdot d \tau_{1} d \tau_{2}, \\
& C_{n_{1} n_{2}}=\frac{1}{\pi^{2}} \int_{0}^{\pi} \int_{0}^{2 \pi} \Gamma\left(\tau_{1}, \tau_{2}\right) \cos n_{1} \tau_{1} \cdot \sin n_{2} \tau_{2} \cdot d \tau_{1} d \tau_{2}, \\
& D_{n_{1} n_{2}}=\frac{1}{\pi^{2}} \int_{0}^{2 \pi} \int_{0}^{2 \pi} \Gamma\left(\tau_{1}, \tau_{2}\right) \cdot \sin n_{1} \tau_{1} \cdot \sin n_{2} \tau_{2} \cdot d \tau_{1} d \tau_{2},
\end{aligned}
$$

Da im vorliegenden Falle die Function gerade ist, verschwinden die ungeraden Glieder der Reihe und nimmt dieselbe mit Vertauschung der Buchstaben $B$ und $D$ die einfachere Form an:

(28) $\Gamma\left(\tau_{1}, \tau_{2}\right)=\frac{1}{4} A_{0,1}+\frac{1}{3} \sum_{1}^{\infty} A_{n_{1} 0} \cos n_{1} \tau_{1}+\frac{1}{3} \sum_{1}^{\infty} A_{0 n_{2}} \cos n_{2} \tau_{2}$

$$
+\sum_{1}^{\infty} n_{1} \sum_{1}^{\infty}\left(A_{n_{1} n_{2}} \cos n_{1} \tau_{1} \cdot \cos n_{2} \tau_{2}+B_{n_{1} n_{2}} \sin n_{1} \tau_{1} \cdot \sin n_{2} \tau_{2}\right)
$$

worin:

$$
\left\{\begin{array}{l}
A_{n_{1} n_{2}}=\frac{1}{\pi^{2}} \int_{0}^{2 \pi} \int_{0}^{2 \pi} r\left(\tau_{1}, \tau_{2}\right) \cdot \cos n_{1} \tau_{1} \cos n_{2} \tau_{2} \cdot d \tau_{1} d \tau_{2}, \\
B_{n_{1} n_{2}}=\frac{1}{\pi^{2}} \int_{0}^{2 \pi} \int_{0}^{2 \pi} \Gamma\left(\tau_{1}, \tau_{2}\right) \cdot \sin n_{1} \tau_{1} \cdot \sin n_{2} \tau_{2} \cdot d \tau_{1} d \tau_{2} .
\end{array}\right.
$$

Zur Darstellung der zweiten am Schluss des \$ 5 erwähnten Functionen würde die allgemeinere, nicht gerade Reihe dienen.

In die Coefficienten (29) sollen jetzt an Stelle der Integrationsvariablen $\tau_{1}, \tau_{2}$ die Variablen $u_{1}, u_{2}$ aus $\S 2$ eingeführt werdeu. Dies geschieht unter Vermittlung der Variablen $t_{1}, t_{2}$, deren Zusammenhang mit $u_{1}, u_{2}$ einerseits und $\tau_{1}, \tau_{2}$ anderseits durch die Formeln (15) und (25) gegeben wird. Für die verschiedenen Functionaldeterminanten zwischen diesen Variablen hat man:

della A Accademia dei Lincei, ser. III, vol. 4 (1879) and rol. 8 (1880); ferner Weierstrass, Abhandlungen zur Functionenlehre, Berlin 1886, S. 160. 


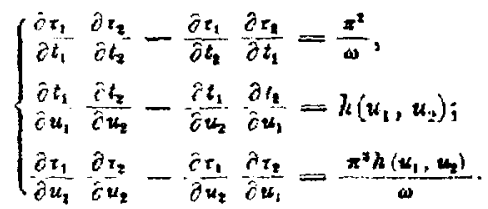

Die dritte dieser Determinanten hat, wie $h\left(u_{1}, u_{1}\right)$, für alle reellen Werthe von $u_{1}, u_{2}$ das nāmliche Vorzeichen, und zwar ist sie positiv, da $h\left(u_{1}, u_{2}\right)$ und w nach (26) gleiches Vorzeichen haben. Führt man daher unter dem Doppelintegral $A_{w_{1} w_{4}}$ in (29) die Variablen $w_{1}, w_{2}$ ein, so erhält man zunächst ohne Rüebsicht auf die Grenzen:

$$
\begin{aligned}
& A_{n_{1} x_{2}}=\frac{1}{\omega} \iint H\left(u_{1}, u_{2}\right) \cdot h\left(u_{1}, u_{1 .}\right) \\
& \cdot \cos \left(n_{1}-\frac{\left.u_{1}\left(u_{1}, u_{2}\right) \cdot \omega_{n}-\psi_{2}\left(u_{1}, u_{2}\right) \cdot \omega_{12} x\right)}{\omega}\right. \\
& \cdot \cos \left(n_{2} \frac{\omega_{11} \psi_{2}\left(u_{1}, u_{2}\right)-\omega_{21} \psi_{1}\left(u_{1}, u_{2}\right)}{\omega} x\right) d u_{1} d u_{2} .
\end{aligned}
$$

Hier sind $H\left(u_{1}, u_{2}\right)$ und $h\left(u_{1}, u_{3}\right)$ bekannte eindeutige Fuuctioneu von $u_{1}, u_{2}$, letztere in (13), erstere in (6) und (14) eingefubrt. Ferne: sind $\psi_{1}\left(u_{1}, u_{2}\right)$ und $\psi_{2}\left(u_{1}, u_{2}\right)$ eindeutige Functionen von $u_{1}, u_{2}$, welche nach (16) und (17) durch Quadratur berechnet werden können. Der Coefficient von $d u_{1}, d u_{2}$ unter dem Doppelintegral ist überdies eime doppelt periodische Fuuction der Variablen $u_{1}, u_{2}$ mit den Periodenparen $2 \pi, 0$ und $0,2 \pi$, wie mit Beugg auf $H\left(u_{1}, u_{2}\right)$ und $h\left(u_{1}, u_{2}\right)$ in $\$ 2$ bemerbt worde und mit Bezug auf das Product der beiden Cosinus aus (20) herrorgeht.

$\mathrm{D}_{\mathrm{m}}$ dje Grenzen des Doppelintegrals (31) zu bestimmen, bemerkt man zuerst, dass das ursprüngliche Integral (29) aber ein Periodenparallelogramm der Function $\Gamma\left(\tau_{1}, \tau_{2}\right)$ in der Ebene der Coordinaten $\tau_{1}, \tau_{2}$ auszudehnen war; die Ecken desselben sind $\tau_{1}, \tau_{2}=0,0 ; 0,2 \pi$; $2 \pi, 0 ; 2 \pi, 2 \pi$. Durch die Substitution (25) wird dieses Parallelogramm auf ein Parallelogramm in der Ebene der Coordinaten $t_{1}, t_{2}$ abgebildet mit den Eckpunkten $t_{:}, t_{2}=0,0 ; 2 \omega_{11}, 2 \omega_{21} ; 2 \omega_{12}, 2 \omega_{22} ; 2 \omega_{11}+2 \omega_{13}$, $2 \omega_{21}+2 \omega_{22}$. Diesem Parallelorramm aber entspricht wieder cin Parallelogramm der Ebene der Coordinaten $u_{1}, u_{2}$ mit den Ecken $u_{1}, u_{1}=0,0 ; 0,2 x ; 2 \pi, 0 ; 2 \pi, 2 \pi$. Das Integral (31) ist daher über ein solches Parallelogramm det Ebene $u_{1}, u_{2}$, ein Periodea. parallelogramm der Function unter dem Integral, auszudebnen, wobei es wegen der Periodicitait der bezeichneten Function auf die Gestalt der Conturen des Parallelogramms uicht aukommt. Man wird sie geradlinig annehmen und setzen: 
$(32)$

$$
\begin{aligned}
A_{n_{1} n_{2}}= & \frac{1}{\omega_{0}} \int_{0}^{2 \pi} \int_{0}^{2 \pi} H\left(u_{1}, u_{1}\right) \cdot h\left(u_{1}, u_{2}\right) \\
& \cdot \cos \left(n_{1} \frac{\omega_{22} \psi_{1}\left(u_{1}, u_{2}\right)-\omega_{12} \psi_{2}\left(u_{1}, u_{2}\right)}{\omega} \pi\right) \\
& \cdot \cos \left(n_{2} \frac{\omega_{11} \psi_{2}\left(u_{1}, u_{2}\right)}{\omega}-\frac{\omega_{21} \psi_{1}\left(u_{1}, u_{2}\right)}{\omega} \pi\right) \cdot d u_{1} d u_{2} .
\end{aligned}
$$

Damit ist die Möglichkejt der Berechnung des Coefficienten $\boldsymbol{A}_{\mathbf{n}_{1} \boldsymbol{x}_{2}}$ dargethan. Die der Coefficienten $B_{n_{2} n_{2}}$ und eventuell der Coefficienten $C_{n_{1} n_{2}}, D_{n_{1} n_{2}}$ gestaltet sich dementsprechend.

$$
\S 8 .
$$

Ueber eine Gattung bedingt periodischer Functionen einer reellon Verầnderlichen.

Die zweifach unendiche trigonometrische Reihe (28) des $\$ 7$, als Function von $t_{1}, t_{2}$ betrachtet, schreitet nach den Cosinus und Sinus der Vielfachen der beiden Ausdrücke:

$$
\tau_{1}=\frac{t_{1} \omega_{22}-t_{2} \omega_{12}}{\omega} \pi, \quad \tau_{2}=\frac{\omega_{11} t_{3}-\omega_{31} t_{1}}{\omega} \pi
$$

fort. Setzt man jetat:

so werden diese:

$$
t_{1}=0, \quad t_{2}=t
$$

$$
\tau_{1}=-\frac{\omega_{12}}{\omega} \pi t, \quad \tau_{1}=\frac{\omega_{12}}{\omega} \pi t .
$$

Die Reihe (28) ist, jetzt als Function von $t$ in Allgemeinen nicht meh periodisch, weil in Folge der Voraussetznng $t_{1}=0$ gleichzeitige Aenderungen von $t_{1}$ und $t_{2}$ um $2 \omega_{11}$ und $2 \omega_{21}$ oder $2 \omega_{12}$ und $2 \omega_{2}$ : nicht mehr stattfinden können.

Nimmt man jedoch an, dass zwischen $\omega_{11}$ and $\omega_{12}$ mit zwei posi. tiven oder negativen ganzen Zahlen $m_{1}$ und $m_{2}$ die Relation besteht

$$
m_{1} \omega_{11}+m_{2} \omega_{12}=0
$$

und setzt man ferner:

so folgt zunächst:

$$
m_{1} \omega_{21}+m_{2} \omega_{22}=T,
$$

$$
-\frac{\omega_{12}}{\omega}=\frac{m_{1}}{T^{\prime}}, \quad, \frac{\omega_{31}}{\omega}=\frac{m_{2}}{T}
$$

und wird damit aus (33):

$$
\tau_{1}=\frac{m_{1} \pi t}{T^{t}}, \quad \tau_{2}=\frac{m_{2} \pi t}{T} .
$$


Demnach werden die einzelnen Glieder der trigonomatrischen Reihen periodische Functionen von $t$ mit der Periode $2 T$, und damit dio durch die Reihen dargestellten Functionen $G(0, t)$ selbst periodisehe kunctionen von $t$ mit der Periode $2 T$. Es folgt daher:

Bestehen groischen 3 reellen Variablen $x_{1}, x_{3}, t$ die Gleichusgen:

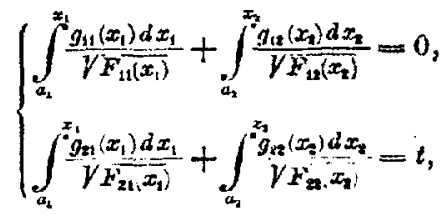

so ist eine beliebig gegebene Function $E\left(x_{1}, x_{9}\right)$ der oberen Intcgralgrenzen $x_{1}, x_{2}$, volche für alle den Ungleichungen $a_{1}<x_{1}<b_{1}$, $a_{2}<x_{2}<b_{2}$ genügendere Werlhepaare $x_{1}, x_{2}$ eindeutig, condlich und sictig ist, unter den in $\$ 1$ angcgebences Bedingningen eine für alle reclles, Werthe von teindeutige, endliche und stetige, sowie bedingt periodische Function von $t$.

Sie ist nämlich im Allgerneinen nicht periolibch, toird aber periodisch mit der Periode

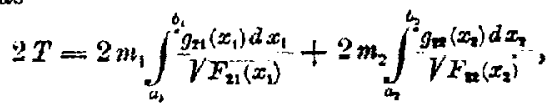

sobald die Bedingung:

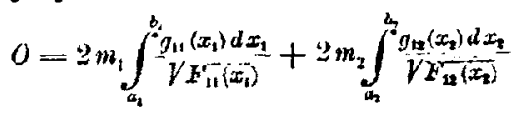

mit irgend welcluen ganzen positiven oder negativen $Z$ ablulen $m_{1}, m_{2}$ erfülle ist.

Analoges würde für die zweite, am Endo von $\$ 5$ angegebene Art von Functionen gelten, nur dass diese unter derselben Bedingung (39) die Puriode $4 T$ bekommen.

Dorpat, im Januar 1887. 